\title{
Robert R. Wilson (1914-2000): the first scientist to propose particle therapy-use of particle beam for cancer treatment
}

\author{
Masahiro Endo 1
}

Published online: 20 October 2017

(C) Japanese Society of Radiological Technology and Japan Society of Medical Physics 2017

\section{The life of Robert R. Wilson}

Robert R. Wilson (Fig. 1) was the first scientist to propose particle therapy - the use of particle beams for the treatment of cancer, which has since been used worldwide, primarily in developed countries.

Robert R. Wilson was born in Wyoming on March 4, 1914 [1]. During his early life, he was relocated a few times but spent most of his life living on ranches where horses and cows were raised. Wilson enrolled at the University of California, Berkeley (UCB) in 1932 and was awarded a Bachelor of Arts degree in 1936. During graduate studies, he joined Ernest O. Lawrence's Radiation Laboratory, which is currently named the Lawrence Berkeley National Laboratory (LBNL). The Radiation Laboratory was becoming a top physics research institute in the United States that later produced several Nobel Prize winners. Wilson worked on cyclotron research under the guidance of E. O. Lawrence and was awarded a Doctor of Philosophy (PhD) degree in 1940.

After obtaining his $\mathrm{PhD}$, Wilson relocated from the UCB to Princeton University to work with Henry D. Smyth. Wilson was involved in Smyth's project developing an electromagnetic separation method for uranium-235 from uranium-238. The separation of uranium isotopes was an important step in the production of the atomic bomb. During 1941, Wilson produced a prototype of an isotopeseparating device known as an "isotron", which used a radiofrequency electric field to separate uranium instead of

Masahiro Endo

endo.masahiro@antm.or.jp

1 Association for Nuclear Technology in Medicine, Tokyo, Japan the static magnetic field used previously, and which was considered more efficient than the previous methods.

Science and technology played an important role in World War II, which began in 1939. In response to Germany's research on the atomic bomb, the United States started the Manhattan Project in 1942, which mobilized scientists from institutions across the United States with the aim of producing atomic bombs. Los Alamos National Laboratory, of which Robert Oppenheimer was the director, had a central role in this project by leading the construction of the bombs. Wilson, along with several Princeton colleagues, joined the Manhattan Project in 1943.

At Los Alamos, Wilson was the leader of the Cyclotron Experiment Group and worked on the use of the cyclotron for measuring the neutron cross section of plutonium. The cyclotron which he used had been relocated from Harvard University. Wilson was in his 20 s at the time and was the youngest leader of an experiment group in the Manhattan Project. When the laboratory was reorganized in August 1944 to focus on the development of an implosion-type nuclear weapon, Wilson became a head of research divisions and supervised four research groups. One of the scientists who worked for Wilson was Emilio Segrè, who later received a Nobel Prize. Although the Manhattan Project lost its initial objective after the surrender of Germany, it was continued and culminated in the bombing of Hiroshima and Nagasaki.

Wilson deeply regretted his participation in the Manhattan Project, which prompted him to develop a keen interest in the peaceful uses of nuclear energy. A study on particle therapy published by Wilson in 1946 was thought to be influenced by this renewed interest.

After the war, Wilson was appointed as an associate professor at Harvard University, but spent most of 1946 at Berkeley designing a new 150-MeV cyclotron for Harvard 


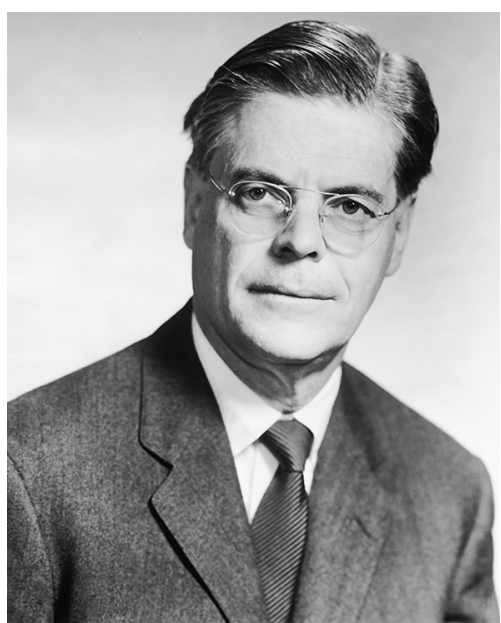

Fig. 1 Robert R. Wilson (Credit: Fermilab.)

University to replace the cyclotron that had been relocated to Los Alamos during the war. Wilson found that highenergy protons produced by the $150-\mathrm{MeV}$ cyclotron could be used for particle therapy. In the same year of 1946, he published an article in the medical journal Radiology titled "Radiological Use of Fast Protons", based on the ideas he had developed at Berkeley [2]. This study was the first to propose the use of particle beams for cancer treatment.

In 1947, Wilson moved to Cornell University and worked as a professor and director of a newly established laboratory for nuclear research. At Cornell, Wilson and his colleagues constructed four electron synchrotrons. The first, a $300-\mathrm{MeV}$ unit, was already being constructed when he arrived. Wilson initiated the construction of a $1.4-\mathrm{GeV}$ synchrotron in 1952, and the last synchrotron built at Cornell was a $12-\mathrm{GeV}$ unit, which remains in use today as the injector for the Cornell Electron Storage Ring (CESR).

In 1967, Wilson assumed the directorship of the new National Accelerator Laboratory which was to be built in Batavia, Illinois. This laboratory was established for constructing of the largest particle accelerator in the world. Under Wilson's talented leadership with mentorship by Lawrence and Robert Oppenheimer, the facility was completed on time and under a budget of 250 million U.S. dollars.

The accelerator was 4 miles in circumference and capable of accelerating protons to $400 \mathrm{GeV}$. Wilson also used this accelerator to initiate the design of the Tevatron, which was capable of accelerating protons to $1 \mathrm{TeV}$. The National Accelerator Laboratory was renamed the Fermi National Accelerator Laboratory in 1974 in honor of Enrico Fermi, a professor at the University of Chicago. This facility is frequently referred to as "Fermilab."

Wilson received many awards and honors, including a Department of Energy Enrico Fermi Award in 1984. Wilson died on January 16, 2000, in Ithaca, New York, at the age of 85 .

\section{Particle therapy-use of particle beams for cancer treatment}

After the war, in 1946, Wilson worked primarily at the Radiation Laboratory (currently the Lawrence Berkeley National Laboratory) in Berkeley, designing a $150-\mathrm{MeV}$ cyclotron. During that time, E. O. Lawrence, the director of the laboratory, asked Wilson to perform radiation shielding calculations for proton beams [3]. During this work, Wilson concluded that the Bragg peak formed by a proton beam in a medium could be used for radiation therapy in the treatment of cancers, and he published a series of analytical results on this subject in the medical journal Radiology. The Radiation Laboratory consisted not only of nuclear physics departments but also of other research departments, of which the Donner Laboratory conducted biological and medical research. John H. Lawrence (Ernest O. Lawrence's younger brother), the director of the Donner Laboratory, had obtained an M.D. degree from Harvard University and was one of the founders of nuclear medicine. He is also known, along with Robert Stones, for having used neutron beams from a cyclotron to perform the world's first neutron therapy before the World War II. This multi-disciplined environment prompted Wilson's interest in cancer treatment and was thought to have inspired him to write his paper on radiotherapy applications of particle beams.

Wilson states his objective in the opening paragraph of his paper, which is paraphrased here: "Particles accelerated by accelerators have not been used in therapy to date because of the small penetration depth of these particles in tissues. However, recently designed accelerators produce high-energy particles capable of reaching any part of the human body. It must have occurred to many people that the particles now become of considerable therapeutic interest. The objective in this paper is to inform medical and biological researchers about the physical properties and utility of particle beams. (Italic font indicates original text)".

In this paper, Wilson first described the qualitative properties of proton beams. Protons usually traverse tissues in a straight line, and the dose (i.e., the kinetic energy imparted to tissues by protons per unit distance) is inversely proportional to the proton kinetic energy, so that protons yield their maximum dose immediately before stopping. The maximum dose is far greater than the dose received at the site of incidence on the skin. Using a diagram-based approach, he introduced the graph in Fig. 2, which demonstrates that the dose peak (known as the Bragg peak), formed immediately before stopping, could provide a large dose to targeted cancers located deep in the body. In the study, he examined, in a semi-quantitative manner, the range straggling and multiple scattering that 


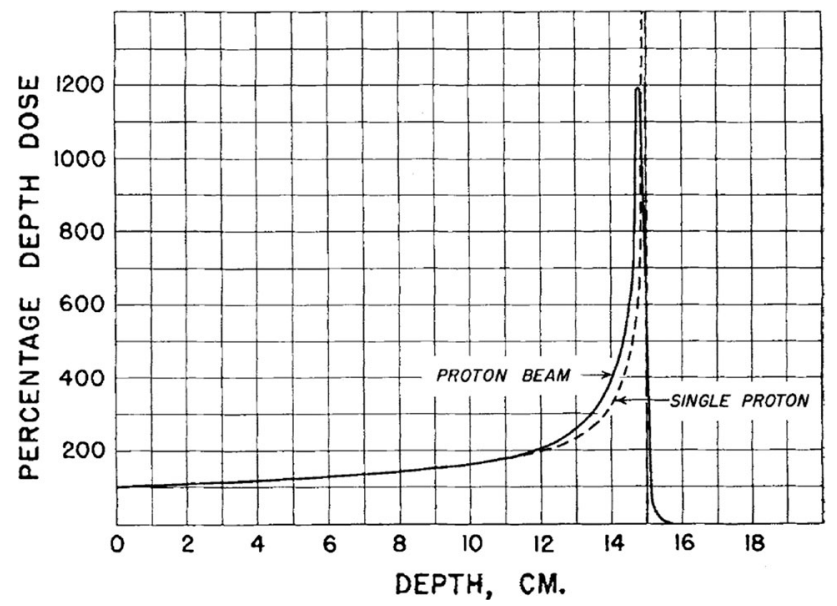

Fig. 2 Dotted curve shows the relative dose due to a single $140-\mathrm{MeV}$ proton. The full curve shows qualitatively the depth dose curve for a beam of 140-MeV proton in tissue (Wilson RR. Radiological use of fast protons. Radiology 1946;47:487-491.)

occurs from proton and tissue interactions, and that do not significantly alter the dose distribution. He concluded that it is possible to administer in a volume of $1.0 \mathrm{cc}$ a dose corresponding to several times the dose applied to the surrounding tissue.

In the paper, Wilson also discussed the therapeutic applications of deuterons, helium ions, and carbon ions, which are heavier than protons and are expected to have an even greater therapeutic impact because of the stronger biological effect. The rationales of particle therapy described in this paper remain valid, although more than 70 years have passed, since it was published.

\section{Berkeley and Harvard}

\subsection{Berkeley}

The research into the therapeutic application of particle beams proposed by Wilson started at the Berkeley Radiation Laboratory. John Lawrence and Cornelius A. Tobias, who had studied aerospace medicine, performed fundamental research on the physics and biology of cancer treatments by using protons, deuterons, and helium ions generated in an 184-inch cyclotron developed in 1948 [4], and they began clinical studies in 1954 [5]. However, in these clinical studies, they did not use particle beams to treat malignant tumors, but irradiated the pituitary gland to destroy its hormone-producing capability and thus treat diseases caused by the abnormal production of hormones by the pituitary gland (e.g., acromegaly). An important reason for targeting the pituitary gland was that diagnostic imaging equipment and other related technologies were at an early stage of development and not at the level of precision needed for the use of particle beams, which limited the assessment of tumor spread. The pituitary gland is located in the sella turcica at the bottom of the skull, and the location and size of this gland are easily identified by orthogonal X-ray imaging. The pituitary gland was first irradiated with $340 \mathrm{MeV}$ protons and then-after an increase in cyclotron energy - with $910 \mathrm{MeV}(227.5 \mathrm{MeV} /$ u) helium ions. Irradiation was performed with use of the plateau region of particle beams with a cross-firing technique, because no traditional method could determine the range of the beam penetrating the skull, which restricted the matching of the position of the Bragg peak with the pituitary gland.

The development of diagnostic imaging equipment in the 1970s, such as the emergence of computed tomography (CT), and the advances in radiotherapy planning with computers allowed the treatment of malignant tumors with particle beams. A method developed at Berkeley allowed the assessment of tumor spread by CT, and it expanded the Bragg peak on the basis of this assessment. In 1976, clinical studies on helium-ion therapy for malignant tumors were started [5]. The BEVALAC accelerator (Fig. 3), which connected the Super HILAC heavy-ion linear accelerator to the Bevatron proton synchrotron to accelerate various ions to high-energy levels, was also completed in this period [6] and started to be used in clinical studies involving $670 \mathrm{MeV} / \mathrm{u}$ neon-ion beams generated by the BEVALAC (Fig. 4). Clinical studies with the 184-inch cyclotron and BEVALAC ended in 1985 and 1992, respectively, with the shutdown of these accelerators. During their operation, these two accelerators together were used for the treatment of 30 patients with proton beams, 2054 patients with helium-ion beams, and 433 patients with neon and other ions [7]. Approximately 50\% of the treatments with helium-ion beams consisted of pituitary gland irradiation and other treatments of benign diseases. In addition to neon-ion treatments, a few other treatments were performed by use of carbon and silicon ions.

\subsection{Harvard}

At Harvard University, the $150-\mathrm{MeV}$ cyclotron that Wilson helped design (Fig. 5) was completed in 1949. The cyclotron could not steadily accelerate protons to energies higher than $110 \mathrm{MeV}$ because of problems related to the radiofrequency acceleration system. However, after modifications in 1955, the cyclotron consistently accelerated protons to energies of up to $165 \mathrm{MeV}$. Similar to those at Berkeley, medical applications at Harvard began in 1962 with pituitary gland irradiations. Because shorter-range $160-\mathrm{MeV}$ proton beams were used for this purpose, the treatments at Harvard were performed with use of a slightly enlarged Bragg peak region instead of the plateau region. 


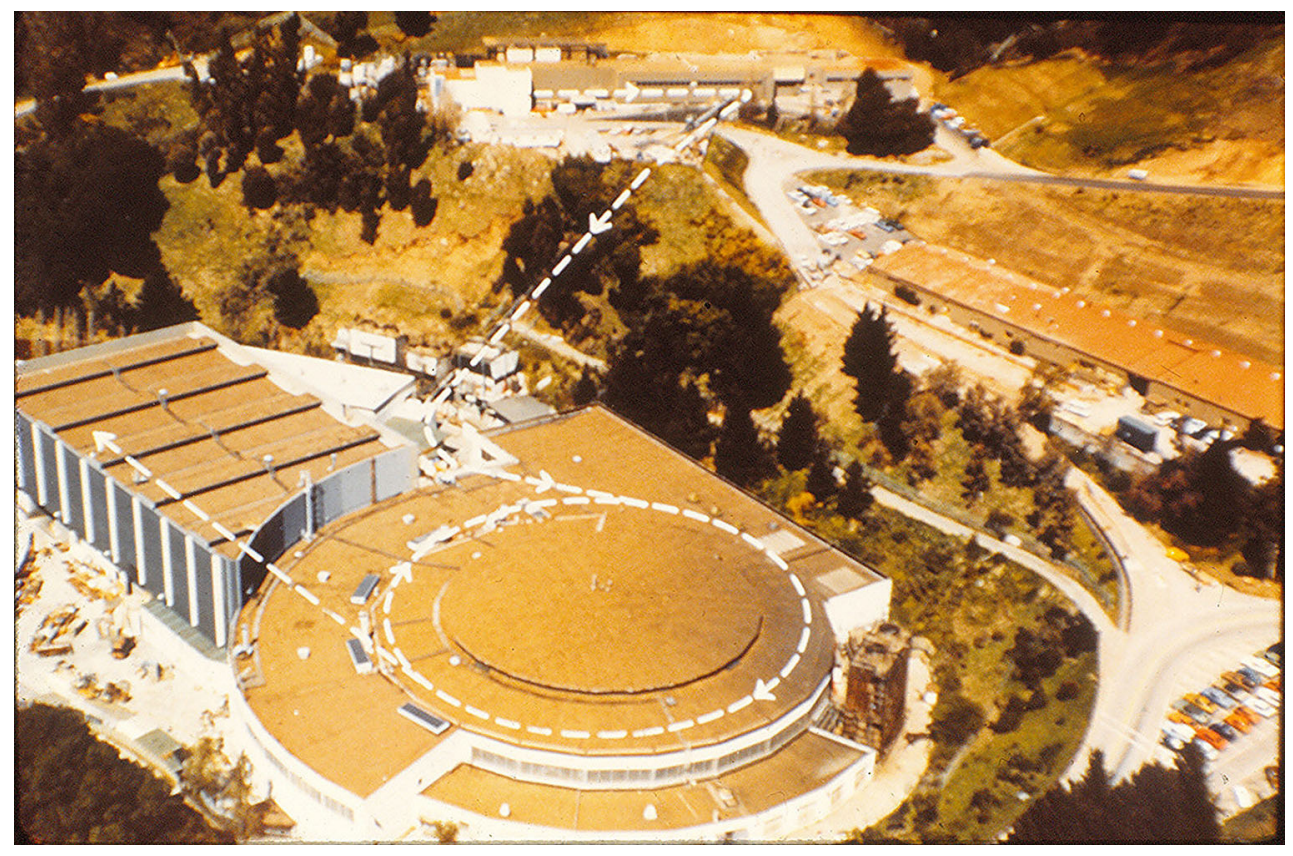

Fig. 3 BEVALAC. Heavy ions are accelerated to a high-energy level by connecting the Super HILAC heavy-ion linear accelerator to the Bevatron proton synchrotron. Particle therapy with neon ions was

Herman D. Suit was the director of the newly established Department of Radiation Medicine at Massachusetts General Hospital (MGH) in 1972. He was a strong proponent of particle therapy for malignant tumors, and in February 1974, the first such treatment, for a posterior pelvic sarcoma, was performed [8], and this kind of treatment was followed at Berkeley later. Michael Goitein, a medical physicist employed by Suit, developed a CT-based $3 \mathrm{D}$ treatment-planning system as well as a patient setup method by registration of X-ray images and digitally reconstructed radiographs (DRR) as positional aid, and, in so doing, introduced treatment systems that were extremely advanced for the time. Similar treatment systems were developed independently at Berkeley.

Radiotherapy systems developed at Harvard and Berkeley were adopted not only for particle therapy, but also for photon therapy, and became the prototype for the high-precision radiotherapy systems used today. A total of 9116 patients were treated with proton therapy at Harvard in the 40-year period before this treatment program was transferred to the newly constructed Proton Therapy Center at MGH in 2002 [7].

\section{Development of dedicated accelerators}

Particle therapy for cancer was started at Berkeley and Harvard in the mid-1970s. When this treatment produced the expected results, in developed countries, including conducted together with nuclear physics research (C) 2010 The Regents of the University of California, through the Lawrence Berkeley National Laboratory.)

Japan, the demand increased for the development of dedicated accelerators instead of sharing accelerators with nuclear research groups. The first dedicated accelerators were a proton therapy system implemented at Loma Linda University in the United States and the Heavy Ion Medical Accelerator in Chiba (HIMAC), located at the National Institute of Radiological Sciences (NIRS) in Japan.

\subsection{Loma Linda University}

Harvard University intended to develop a dedicated accelerator for proton therapy in the 1980s, but this milestone was first achieved at Loma Linda University in Southern California. In 1985, James M. Slater, the chairman of the Department of Radiation Medicine at the Loma Linda University Medical School, commissioned Fermilab to develop a small-scale proton accelerator suitable for a hospital [9]. With the encouragement of Robert Wilson, who had already retired as director, Fermilab developed a synchrotron capable of accelerating a proton beam to $250 \mathrm{MeV}$ (Fig. 6), which was installed at Loma Linda University. In contrast to the previous systems that used a fixed beam, this system contained a rotating gantry that allowed irradiation of all parts of the body in any direction. Since the development of this system, a rotating gantry has become a standard feature of proton therapy systems. The proton therapy system at Loma Linda was used initially for the treatment of patients at the end of 1990 and treated a total of 18,362 patients (the largest number of patients 


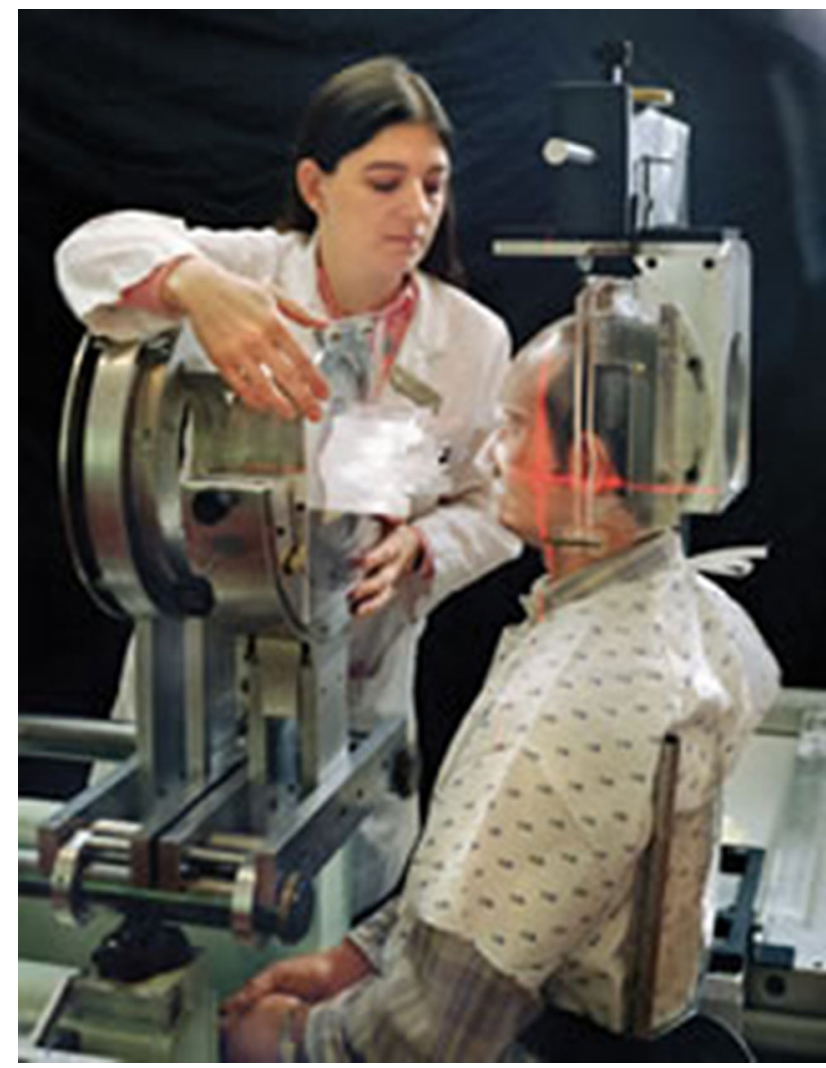

Fig. 4 Treatment at the BEVALAC. The patient was irradiated with a neon-ion beam on the left side and immobilized with a transparent head mask. The treatment site was determined precisely with use of laser pointers and X-ray imaging. A compensating filter and collimator were installed at the end of the port to form the beam shape for covering the tumor (@ 2010 The Regents of the University of California, through the Lawrence Berkeley National Laboratory.)

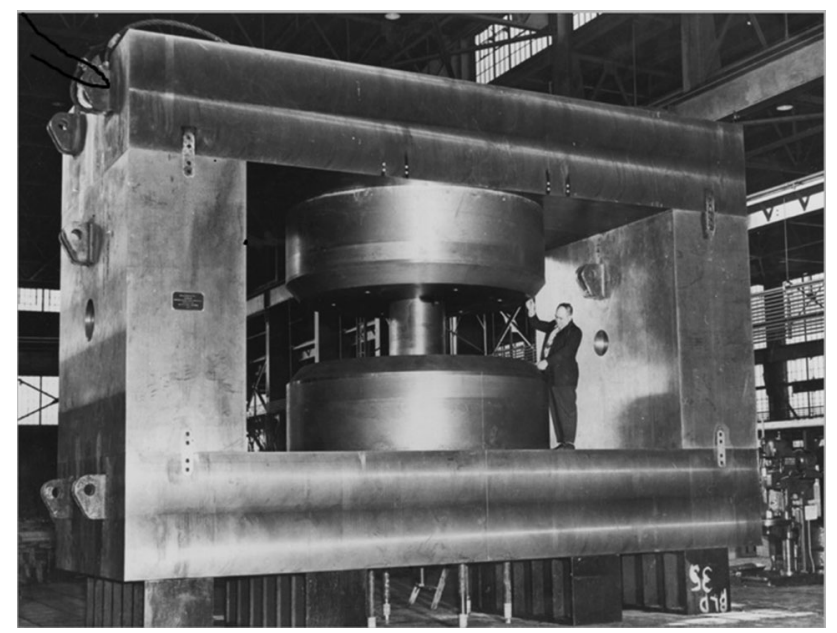

Fig. 5 Iron core of the electromagnet of the $150-\mathrm{MeV}$ cyclotron. The two central disks were the magnetic poles, and the external frame was the yoke confining the magnetic flux. A spacer was placed between the magnetic poles to maintain a precise distance between them. The magnets were assembled by Albert (Pop) Poperell with his special crew from Bigge Drayage Co. of California, as written up in the Boston Globe on January 11th, 1948

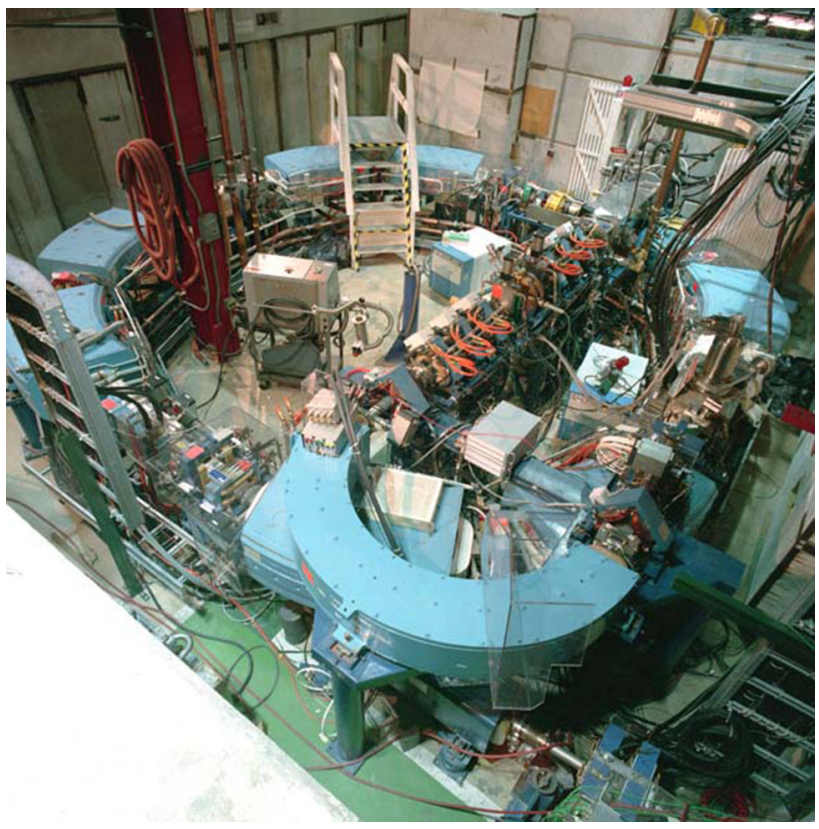

Fig. 6 250-MeV synchrotron being assembled and adjusted at Fermilab. After completion, the synchrotron was transported to Loma Linda University and used for proton therapy (Credit: Fermilab.)

treated in the world's particle therapy facilities) over a 25-year period until the end of 2015 [7]. The system remains in operation at the time of this writing and continues to update treatment records on a daily basis.

\subsection{HIMAC at NIRS}

The LBNL intended to start performing ion beam therapy with a dedicated accelerator in the late 1970s, but was unable to carry out this plan because of lack of funding. The first facility in the world to build a dedicated ion accelerator and to start treating patients was the National Institute of Radiological Sciences (NIRS) in Japan. In the 1970s, fast-neutron therapy was performed at the NIRS with use of a cyclotron. In addition, the first proton therapy in Japan was conducted there, although the proton energy was only $70 \mathrm{MeV}$ and its targeted disease were quite limited. At the beginning of the 1980s, the NIRS started preparing for its next project, which involved heavy-ion therapy. Design studies began in 1984, and the construction of a heavy-ion synchrotron designated as Heavy Ion Medical Accelerator in Chiba (HIMAC) (Fig. 7), began in 1987 with the appointment of Yasuo Hirao from the Institute for Nuclear Study, University of Tokyo, as the project leader [10]. The HIMAC was completed in 1993. It was capable of accelerating various ions, from helium to neon, but used $400 \mathrm{MeV} / \mathrm{u}$ carbon ions. These took the place of the neon ions used at the LBNL. Carbon ions were chosen, because they allow the maximization of the 


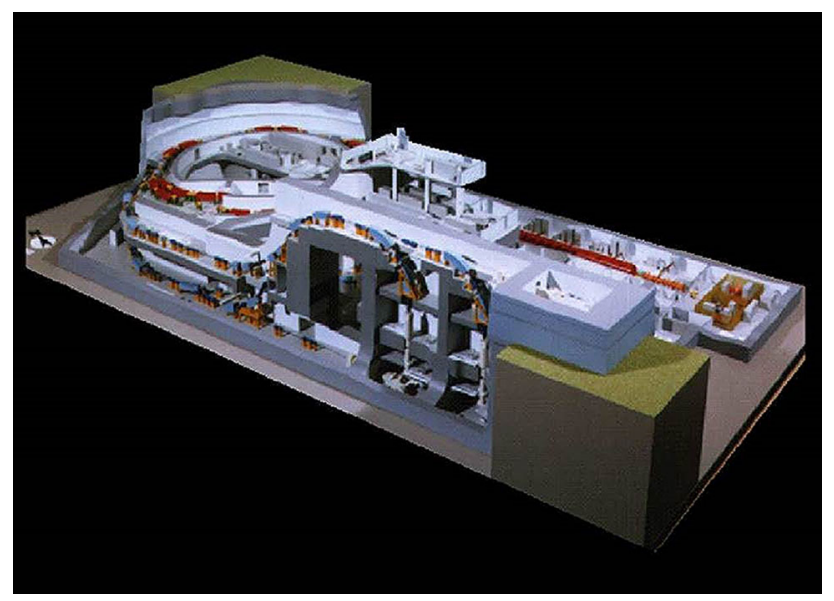

Fig. 7 Model showing NIRS's HIMAC. The HIMAC consists of an injector, a two-stage synchrotron, three therapeutic irradiation rooms, and experimental irradiation rooms

biological dose applied to deep-seated tumors relative to the dose applied to the surrounding healthy tissue. Carbon ions were used at the heavy-ion therapy facilities, those followed HIMAC, based on the results of fundamental and clinical research conducted with use of the HIMAC. Heavy-ion therapy with the HIMAC was initiated in 1994 and, with ongoing improvements to the HIMAC, continues to this day. The HIMAC treated 10,486 patients (the second largest number of treated patients in the world's particle therapy facilities) over a 22-year period until the end of 2015 [7].

The number of particle therapy facilities has increased rapidly in recent years. Approximately 100 facilities are currently either in operation or under construction throughout the world; this number is expected to increase in the future. As covered in this report, Wilson's initial proposal marked the beginning of a substantial trend in research and practical applications. The significance of his proposal cannot be overemphasized.

\section{References}

1. Wikipedia. Robert $\mathrm{R}$ Wilson. https://en.wikipedia.org/wiki/ Robert_R._Wilson. Accessed 30 Aug 2017.

2. Wilson RR. Radiological use of fast protons. Radiology. 1946;47:487-91.

3. The promise of ion beam cancer therapy. http://newscenter.lbl. gov/2010/10/18/ion-beam-therapy/. Accessed 30 Aug 2017.

4. Tobias CA, Anger HO, Lawrence JH. Radiological use of high energy deuterons and alpha particles. Am J Roentgenol. 1952;67:1-27.

5. Boone ML, Lawrence JH, Connor WG, et al. Introduction to the use of protons and heavy ions in radiation therapy: historical perspective. Int J Radiat Oncol Biol Phys. 1977;3:65-9.

6. Tobias CA. Pretherapeutic investigations with accelerated heavy ions. Radiology. 1973; 108:145-58.

7. Particle Therapy Co-Operative Group. Particle therapy patient statistics (per end of 2015). https://www.ptcog.ch/archive/ patient_statistics/Patientstatistics-updateDec2015.pdf. Accessed 30 Aug 2017.

8. Wilson R. A brief history of the Harvard University cyclotrons. http://users.physics.harvard.edu/ wilson/cyclotron/history.html. Accessed 30 Aug 2017.

9. Robert R. Wilson: Remembered as "Father of Proton Therapy" and achievements in physics and medicine. http://www.protontherapy.org/pr10.htm. Accessed 30 Aug 2017.

10. Hirao Y, Ogawa H, Yamada S, et al. Heavy ion synchrotron for medical use-HIMAC project at NIRS, Japan. Nucl Phys. 1992;A538:541c-50c. 